\title{
Effects of Vegetarian Diets on Aging and Longevity
}

\author{
Paul Walter
}

Vegetarian diets are very heterogeneous, as are their effects on nutritional status, health, and longevity. They encompass not only various animal food avoidance patterns but also differing health-related attitudes as well as widely philosophical and religious beliefs. Vegetarianism is often considered to be a special lifestyle, because many vegetarians are nonsmokers, drink little if any alcohol, and engage in more than average physical exercise. When we evaluate results with vegetarians, we therefore not only have to take into account the uncertainty concerrfing the compliance with their diets but also many confounding factors due to the different life patterns.

A vegetarian is usually defined as someone who does not eat animal flesh (meat, poultry, fish). A strict vegetarian, or vegan, specifically excludes all animal products including dairy products and eggs, and the diet therefore consists mainly of plant foods such as fruits, vegetables, legumes, grains, seeds, and nuts. The lactovegetarian diet includes dairy products, whereas the lacto-ovovegetarian diet includes dairy products and eggs. Even within the classification of these diets, considerable differences may exist in the choice of plant foods. The "New Vegetarians" in The Netherlands and the United States, for example, are followers of the teachings of Ohsawa, ${ }^{1}$ preferring natural unprocessed foods, which he called macrobiotics, consisting of relatively large amounts of brown rice accompanied by smaller amounts of fruits; followers of the veganfruitarian diet restrict their diet to raw fruits, nuts, and berries.

Many studies of vegan diets have been carried out with Seventh Day Adventist vegetarians. For idealistic reasons, this group shows good compliance with their diet and these results are therefore given considerable importance in the literature. However, the originally strict diet limitations of this group have been changing and the American Adventists of today are mainly lactovegetarians, whereas in Germany they consume a mixed diet but one that contains a lot of fruit and vegetables.

Concerning the nonvegan diets, it has been very difficult to attribute the results to certain subgroups such as

Professor Walter is in the Biochemistry Department, University of Basel, Basel, Switzerland. lacto- or lacto-ovovegetarians. The fat composition of the diet, in particular, may vary greatly depending on whether the fat originates from dairy products or from plants.

There are a few good reviews in the literature ${ }^{2,3}$ covering mainly the period from 1960 through 1990, when manystudies were performed. Since 1990, many studies have been in progress that concern more specific effects of vegetarian diets on human health. They deal with the risks on the one hand and with the beneficial effects on the other. Both are important for longevity. We know that good nutrition during growth is an important basis for our health when we get older, and therefore nutritional risks should be avoided, especially during the early period of our life. But after the growing period, nutrition remains a crucial factor for maintenance of good health and prevention of disease in later years. Many recent studies give strong evidence - independent of meat consumption - for an inverse relationship between vegetable/fruit intake and various types of cancer, for a relationship between type of dietary fat and coronary heart disease (for review, see Willett ${ }^{4}$ ), and for the effect of dietary fiber intake on mortality from coronary heart disease, cancer, and other sources. ${ }^{5}$ Because these nutritional aspects are directly related to vegetarian diets, it seems reasonable to include them in the discussion as well.

\section{Diet Adequacy and Risks of Vegetarian Diets}

In principle, protein from plant sources alone can provide adequate amounts of the essential amino acids, provided that the dietary protein sources are reasonably varied, e.g., maize with a protein low in lysine should be combined with beans that are high in this amino acid. Soya protein has been shown to be nutritionally equivalent to proteins of animal origin and thus can serve as a main source of protein intake. The vegetarians who accept dairy products and eggs, of course, have no problem obtaining adequate protein supplies, whereas vegans have to be especially careful to combine various plant proteins in order to avoid a deficiency of a single one of the essential amino acids. Actually, as recently summarized by Margetts and Jackson, ${ }^{6}$ the adult requirement for protein in the diet is again under debate. Young and Pellet ${ }^{7}$ claim that requirements for essential amino acids cannot be satisfied by diets based 
upon cereals only unless at least $30 \%$ of total protein is derived from animal sources or at least $40 \%$ of the total protein is derived from animal sources plus a meat substitute such as soya.

With regard to a possible deficiency in micronutrients, special attention should be given to vitamins $B_{12}$ and $\mathrm{D}_{3}$ as well as to iron and zinc. Vitamin $\mathrm{B}_{12}$ is present in substantial amounts only in animal foods. Its deficiency is therefore a matter of concern in diets that are partially or totally devoid of animal sources. Especially for vegans there clearly exists a risk of vitamin $B_{12}$ deficiency, as has been reported in the literature..$^{8-10}$

It should also be remembered that depletion of vita$\min B_{12}$ stores in vegans is slow and depends on the initial body vitamin $B_{12}$ pool, and therefore symptoms of vitamin $\mathrm{B}_{12}$ deficiency appear slowly but are nevertheless very dangerous. A special problem, therefore, arises in children of vegan women with low vitamin $B_{12}$ stores where special precautions must be taken. ${ }^{11,12}$

A good deal of confusion exists about how much and what kind of vitamin $B_{12}$ can be supplied by plants and by microbiologic sources. Fermented soya products such as tempeh contain many corrinoids (i.e., ring structures similar to vitamin $\mathrm{B}_{12}$, which have little if any vitamin $\mathrm{B}_{12}$ activity in humans. ${ }^{13}$ Similarly, spirulina, another plant product, also contains corrinoids with virtually no vitamin $B_{12}$ activity. A recent article ${ }^{14}$ reported that vegans who consumed nori or chlorella seaweed had vitamin $B_{12}$ concentrations in the serum twice as high as those who did not eat the seaweed. However, these values were still significantly lower than normal. It can therefore be concluded that in the long run all these products cannot sufficiently replace vitamin $\mathrm{B}_{12}$ from animal sources. Vegans as well as other vegetarians therefore are advised to consider supplementing their diet with vitamin $B_{12}$, e.g., by including processed food that is fortified with this vitamin.

It should be noted that the standard microbiologic assay for vitamin $B_{12}$ in foodstuffs cannot differentiate between inactive plant vitamin $B_{12}$ derivatives and vitamin $B_{12}$ from animal sources. Therefore, results in the literature on vitamin $B_{12}$ content in plants must be examined carefully.

Many risk groups today have a low folic acid intake. However, on the whole, vegetarians do not seem to have a greater folic acid deficiency problem than nonvegetarians unless they boil their green leafy vegetables at high temperatures. ${ }^{15,16}$

Vitamin $\mathrm{D}_{3}$ deficiency and lack of calcium do not usually occur in lacto-ovovegetarians because of their intake of milk and dairy products. In the United States, although $70 \%$ of calcium uptake is from dairy products, adequate calcium can be supplied for vegans if sufficient amounts of vegetables and fruits high in calcium are consumed. Vitamin $D_{3}$, however, may turn out to be critical for strict vegans. ${ }^{4}$
One of the main problems of vegetarian diets is the danger of iron deficiency and consequent anemia. Iron bioavailability from foods of plant origin is low compared with that from meat. Consequently, vegetarians have a higher risk of iron deficiency than meat eaters. Among vegetarians, who often have a limited intake of heme iron, the effect of vitamin C-containing foods on improving the absorption of nonheme iron is of particular importance. ${ }^{17}$ On the other hand, nonheme iron binds to phytates, tannins, and phosphates, all commonly present in plant food diets. Phytates are commonly found in whole grain, bran, and soya products and have strong inhibitory effects on iron absorption. ${ }^{18}$ Furthermore, oxalic acid, present in spinach, rhubarb, etc., also forms insoluble complexes with iron, thereby inhibiting its absorption. Vegetarians are therefore advised to consume plant foods that are rich in nonheme iron and low in substances that decrease the absorption of this trace element. Special problems arise in children born to vegetarian mothers with lower stores of iron. If these children are breast-fed for a long period they more likely will develop anemia. It also has been reported that low iron status in the first trimester of pregnancy may increase the risk of premature birth. ${ }^{19,20}$ Therefore, additional intake of iron before conception and during pregnancy is necessary for vegetarians.

Zinc deficiency also may be of concern, because in the United States over two-thirds of the zinc supply comes fromanimal products such as red meat, eggs, oysters, and other seafood. Several studies have reported lower concentrations of plasma zinc for vegetarians and vegans compared with nonvegetarians. ${ }^{21-23}$ Whether these slightly lower concentrations of plasma zinc are of clinical significance, however, remains to be investigated.

Concerning the n-3 fatty acids there is some concern, especially for infants who are born to vegan mothers and then breast-fed. It is known that the n-3 fatty acids are important for development of the central nervous system and the retina. Because vegans and other vegetarians do not eat seafood, their uptake of eicosapentanoic acid and docosahexanoic acid is very small. However, the biologic precursor of these two compounds, a-linolenic acid, occurs in some plant oils, and it is possible that endogenous synthesis of the longer chain derivatives may be sufficient. It appears that more research in this area is required before exact conclusions can be drawn. ${ }^{3,24,25}$

If we look at the influence of vegetarian diet on growth and development, the results are equivocal and of course depend on the kind of vegetarian diet consumed., ${ }^{3,11}$ The classic experiment by Widdowson and $\mathrm{McCance}{ }^{26}$ clearly demonstrated that children will grow and develop quite normally on a diet consisting of plenty of bread and vegetables with minimum amounts of milk and meat. However, several studies report that vegan children often fail to grow as well as their omnivorous cohorts (for review, see $A \operatorname{costa}{ }^{27}$ ). Lower rates of growth, particularly in the first 5 
years of life, have been reported for children reared on vegan ${ }^{28}$ and on macrobiotic diets. ${ }^{29} \mathrm{~A}$ critical issue seems to be intake of dietary fiber, which may have a greater negative effect on nutrient and energy balances in infants and children than in adults. ${ }^{27}$ According to Jakobs and Dwyer, ${ }^{30}$ nutritional deficiencies that are rare in the general population have occurred in children reared on vegetarian and vegan diets, and therefore the growing stage seems to be a critical period. The same is true for pregnancy where-as mentioned earlier-vitamin $B_{12}$, vitamin $D_{3}$, iron, and $n-3$ fatty acids may be critical factors for the development of babies of vegan mothers.

\section{Benefits of Vegetarian Diets}

In 1988, Dwyer ${ }^{2}$ reviewed the effects of vegetarian diets on nutritional status, health, and longevity. Her summary points out that mortality rates are similar or lower for vegetarians than for nonvegetarians and that the risks of dietary-deficiency disease are increased for vegan but not for all vegetarian diets. She mentions that both vegetarian dietary and lifestyle practices are involved and that the evidence for decreased risks for certain chronic degenerative diseases varies: data are strong that vegetarians are at lower risk for obesity, constipation, and lung cancer; evidence is good that risks for hypertension, coronary heart disease, type II diabetes, and gallstones are lower; data are only fair to poor that risks of breast cancer, diverticular disease of the colon, colonic cancer, calcium kidney stones, and osteoporosis are lower.

If we look at mortality rates as an index for longevity in more detail, ${ }^{2}$ there is a very large study in which 27,530 vegetarian and nonvegetarian Seventh Day Adventists living in California were followed. ${ }^{31}$ After 21 years of follow-up, those whose food habits were mostly vegetarian were found to have lower age-specific mortality rates than those with more omnivorous eating practices. In this and several other studies with groups of Seventh Day Adventist vegetarians, coronary heart disease mortality rates were lower and cancer rates were no higher than in the general population. ${ }^{2}$ In other studies, lower death rates for cancer and heart disease have been observed in some groups of Seventh Day Adventist vegetarians. ${ }^{32,33}$ However, confounding factors, such as abstinence from smoking, alcohol, tea, and coffee, may have played a role. Actually, Fraser et al. ${ }^{34}$ showed that the lower rate of lung cancer in Seventh Day Adventists could be attributed to abstinence from tobacco. In a recent study, a cohort of 1905 vegetarians and persons living a "healthy country lifestyle" occasionally consuming meat products were followed for 11 years in the Federal Republic of Germany. ${ }^{35}$ So far, 225 deaths have been observed and mortality from all causes was reduced by $50 \%$ compared with the general population. Cancer mortality was reduced by $50 \%$ in men but by only $25 \%$ in women. The deficit in cancer deaths was observed mainly for lung and gastrointestinal cancer in males and for gastrointestinal cancer in females. Deaths from diseases of the respiratory and digestive systems were also reduced by about $50 \%$. However, a slight increase of deaths occurred as a result of anemia. When the strict and moderate vegetarians were analyzed separately, the strongest difference was found for ischemic heart disease, which was more than $50 \%$ less frequent among strict vegetarians for both sexes than for the control group, confirming earlier observations. ${ }^{36}$ Again, confounding factors such as socioeconomic status and smoking are mentioned.

It should especially be emphasized that vegetarians are seldom overweight, as observed in many studies. ${ }^{37-40}$ This is very likely due to the fact that vegetarian diets containing fiber and complex carbohydrates have a lower energy density ${ }^{41}$ and cause satiety at a lower calorie intake level. The fact that overweight and obesity are less frequent in vegetarians may in itself be a reason for greater longevity, because many studies have shown these two parameters to be important determinants for mortality. ${ }^{42-47}$

\section{Final Conclusions}

1) From what we know, vegetarian diets result in a lower risk for many diseases such as obesity, cardiovascular disease, some types of cancer, constipation, hypertension, and type II diabetes. If we take the observed lower mortality rates as a parameter, longevity is higher.

2) People on a vegan diet have an increased risk of iron, vitamin $B_{12}$, vitamin $D_{3}$, and calcium deficiency; eventually also zinc, n-3 fatty acids, and protein intake may not fulfill the basic requirements. Except for vitamin $B_{12}$, it appears that a vegan diet can fulfill the requirements, but it takes quite a bit of knowledge and even expertise to choose the fruits and vegetables that contain all the necessary nutrients.

3) Based on our present knowledge that high contents of vegetables, fruits, and complex carbohydrates and low amounts of saturated fatty acids are correlated with a reduced risk for the same diseases mentioned under point 1 , it is obvious that total abstinence from eating meat is not a major factor for the beneficial effects of vegetarian diets.

4) The vegetarian-type diet with lots of vegetables and fruits and complex carbohydrates can be considered a prudent diet in the sense of today's guidelines (see, e.g., Dietary Guidelines for Americans 1990). ${ }^{48}$

5) History has shown that vegetarians were right when they claimed more than 100 years ago that the vegetarian diet including fruits, vegetables, fibers, and complex carbohydrates is a healthy one.

6) The inclusion of some low-fat meat and fish does not seem to be harmful; it could actually be beneficial in lowering the risk of deficiencies in some extreme cases of vegetarianism.

7) Finally, we should realize that about $40 \%$ of today's world grain production is used for meat-producing livestock. ${ }^{49}$ This conversion of cereal grains and other food 
concentrates to animal products involves large losses in energy; $1 \mathrm{~kg}$ of American beef requires $5 \mathrm{~kg}$ of grain. Therefore, if meat consumption could be lowered, more cereal grains and other valuable food components could be used to improve the world's nutrition.

1. Ohsawa G. Macrobiotics: an invitation to health and happiness. San Francisco: George Ohsawa Macrobiotic Foundation, 1991

2. Dwyer JT. Health aspects of vegetarian diets. Am J Clin Nutr 1988;48:712-38

3. Dwyer JT. Nutritional consequences of vegetarianism. Annu Rev Nutr 1991;11:61-91

4. Willett WC. Diet and health: what should we eat? Science 1994;264:532-7

5. Kromhout D, Bosschieter EB, De Lezenne Coulander C. Dietary fibre and 10-year mortality from coronary heart disease, cancer, and all causes. The Zutphen Study. Lancet 1982;ii:518-22

6. Margetts BM, Jackson AA. Vegetarians and longevity. Epidemiology 1993;4(3):278-9

7. Young VR, Pellet PL. Current concepts concerning amino acids in adults and their implications for international planning. Food Nutr Bull 1990;12:289-300

8. Misra HN, Fallowfield JN. Sub-acute combined degeneration of the spinal cord in a vegan. Postgrad Med J 1971;47:624-6

9. Campbell M, Lofters WS, Gibbs WN. Rastafarianism and the vegan syndrome. Br Med J 1982;285:16178

10. Dagnelie PC, Van Staveren WA, Vergote FJVRA, et al. Increased risk of vitamin B12 and iron deficiency in infants on macrobiotic diets. Am J Clin Nutr 1989;50:818-24

11. Sanders TAB. Growth and development of British vegan children. Am J Clin Nutr 1988;48:822-5

12. Lamberg-Allardt $C$, Kärkkäinen $M$, Seppänen $R$, et al. Low serum 25-hydroxyvitamin $D$ concentrations and secondary hyperparathyroidism in middle-aged white strict vegetarians. Am J Clin Nutr 1993;58:684-9

13. Herbert V, Drivas $G$, Manusselis $C$, et al. Are colon bacteria a major source of cobalamin analogues in human tissues? Trans Assoc Am Phys 1984;97:16171

14. Rauma $A L$, Törrönen $R$, Hänninen $O$, et al. Vitamin $B-$ 12 status of long-term adherents of a strict uncooked vegan diet ("living food diet") is compromised. Am Inst Nutr 1995;2511-5

15. Dwyer JT. Health implications of vegetarian diets. Compr Ther 1983;9(3):23-8

16. Dwyer JT. Vegetarian diets in pregnancy and lactation: recent studies in North Americans. $\mathrm{J}$ Can Diet Assoc 1983;44:26-34

17. Hallberg $L$, Brune $M$, Rossander-Hulthen $L$. Is there a physiological role of vitamin $\mathrm{C}$ in iron absorption? Ann NY Acad Sci 1987;498:324-32

18. Hazell T. Relating food composition data to iron availability from plant foods. Am J Clin Nutr 1988;42:50917

19. Kim I, Hungerford DW, Yip R, et al. Pregnancy nutrition surveillance system-United States, 1979-1990. MMWR Center for Disease Control Surveillance Summaries (United States) 1992;41:25-34

20. Scholl TO, Hediger ML, Fischer RL, Shearer JW. Ane- mia vs iron deficiency: increased risk of preterm delivery in a prospective study. Am $\mathrm{J} \mathrm{Clin} \mathrm{Nutr}$ 1992;55:985-8

21. Anderson BM, Gibson RS, Sabry JH. The iron and zinc status of long term vegetarian women. Am J Clin Nutr 1981;34:1042-9

22. King JC, Stein T, Doyle M. Effect of vegetarianism on zinc status of pregnant women. Am J Clin Nutr 1981;34:1049-55

23. Freeland-Graves J. Mineral adequacy of vegetarian diets. Am J Clin Nutr 1988;48:859-62

24. Sanders TAB, Reddy S. Nutritional implications of a meatless diet. Proc Nutr Soc 1994;53:297-307

25. Reddy S, Sanders TAB, Obeid $O$. The influence of maternal vegetarian diet on essential fatty acid status of the newborn. Eur J Clin Nutr 1994;48:358-68

26. Widdowson EM, McCance RA. Studies on the nutritive value of bread and the effect of flour on the growth of undernourished children. London: MRC Special Report Series, 1954;no. 287

27. Acosta PB. Availability of essential amino acids and nitrogen in vegan diets. Am J Clin Nutr 1988;48:86874

28. Sanders TAB, Manning J. The growth and development of vegan children. J Human Nutr Dietetics 1992;5:11-21

29. Dagnelie $P C$, Van Staveren WA, Van Klaveren JD, Burema J. Do children on macrobiotic diets show catch-up growth? Eur J Clin Nutr 1988;42:1007-16

30. Jacobs C, Dwyer JT. Vegetarian children: appropriate and inappropriate diets. Am J Clin Nutr 1988;43(suppl 3):811-8

31. Kahn RH, Phillips RL, Snowdon DA, et al. Association between reported diet and all cause mortality: twenty-one year follow up on 27,530 adult Seventh Day Adventists. Am J Epidemiol 1984;119:775-87

32. Snowden DA. Animal product consumption and mortality because of all causes combined, coronary heart disease, stroke, diabetes, and cancer in Seventh Day Adventists. Am J Clin Nutr 1988;48:739-48

33. Fonnebo V. Mortality in Norwegian Seventh Day Adventists 1962-1986. J Clin Epidemiol 1992;45:157-67

34. Fraser GE, Beeson WL, Phillips RL. Diet and lung cancer in California Seventh Day Adventists. Am J Epidemiol 1991;133:683-93

35. Chang-Claude J, Frentzel-Beyme R, Eilber U. Mortality pattern of German vegetarians after 11 years of follow-up. Epidemiology 1992;3:395-401

36. Burr ML, Sweetnam PM. Vegetarianism, dietary fiber, and mortality. Am J Clin Nutr 1982;36:873-7

37. Sacks FM, Castelli WP, Donner A, et al. Plasma lipids and lipoproteins in vegetarians and controls. $\mathrm{N}$ Engl J Med 1975;292:1148-51

38. Rottka $H$, Thefeld W. Gesundheit und vegetarische Ernährungsweise. Akt Ernähr 1984;9:209-16

39. Burr ML, Butland BK. Heart disease in British vegetarians. Am J Clin Nutr 1988;48:830-2

40. Beilin LJ, Rouse IL, Armstrong BK, et al. Vegetarian diet and blood pressure levels: incidental or causal association? Am J Clin Nutr 1988;48:806-10

41. Kohlmeier L, Mensink GBM, Hermann-Kunz E. Effects of lifestyle on nutrient requirements. In: Pietrzik K, ed. Modern lifestyles, lower energy intake and micronutrient status. London: Springer-Verlag, 1991:3-19 
42. Dyer AR, Stamler J, Berkson DM, et al. Relationship of relative weight and body mass index to 14-year mortality in the Chicago Peoples Gas Company Study. J Chron Dis 1975;28:109-23

43. Lew EA, Garfinkel L. Variations in mortality by weight among 750,000 men and women. J Chron Dis 1979;32:563-76

44. Hubert HB, Feinleib M, McNamara PM, et al. Obesity as an independent risk factor for cardiovascular disease: a 26-year follow-up of participants in the Framingham Heart Study. Circulation 1983;67:96877

45. Waaler HT. Height, weight and mortality: the Norwegian experience. Acta Med Scand 1984;215:1-56
46. Wilcosky T, Hyde J, Anderson JJB, et al. Obesity and mortality in the Lipid Research Clinics Program follow-up study. J Clin Epidemiol 1990;43:743-52

47. Wienpahl J, Ragland DR, Sidney S. Body mass index and 15-year mortality in a cohort of black men and women. J Clin Epidemiol 1990;43:949-60

48. Anonymous. Nutrition and your health. Dietary guidelines for Americans. 3rd ed. Washington, DC: US Department of Agriculture and US Department of Health and Human Services, 1990

49. McMichael A. Vegetarians and longevity: imagining a wider reference population. Epidemiology 1992;3:389-91

\section{Invited Comment}

Jorge L. Rosado

A large proportion of the population in Mexico consume diets consisting primarily of plant foods. It has been reported that $80 \%$ of Mexicans, representing a significant part of the urban population and most of the less developed rural areas, consume diets based on corn tortillas, beans, vegetables, and fruits. Tortillas and beans provide most of the dietary energy and protein. Inclusion of animal products is occasional and highly variable in both population groups and regions. Because a majority of Mexicans include high amounts of plant foods in their diets, the potential adverse effects as well as the potential benefits of such diets are relevant for the population. This comment has the objective of reviewing some of the potential risks and benefits of vegetarian diets in the Mexican population.

\section{Adverse Nutritional Effects of the Plant-Based Rural Mexican Diet}

Nutrient deficiencies are due to poor ingestion of a nutrient, a decrease in the nutrient bioavailability, and/or an increase in the requirements for the nutrient. All these mechanisms are relevant to understanding the association between nutrition deficiencies and intake of plant-based diets.

Plant-based diets are less energy dense and also may contain less protein. The information of the most recent National Nutrition Survey ${ }^{1}$ carried out in rural areas in Mexico shows that deficient energy and protein intake occurs only in the most marginal regions of the country, among the poorest groups of the population. The inges-

Dr. Rosado is at the Departamento de Fisiología de la Nutrición, Instituto Nacional de la Nutrición Salvador Zubirán, Vasco de Quiroga No. 15, Deleg. Tlalpan, 14000, México, D.F. tion of some vitamins, especially vitamin A, riboflavin, and ascorbic acid, is reported to be low in several groups of Mexicans and is generally associated with the rural Mexican diet. ${ }^{2}$ In general, intake of minerals is not deficient even though iron deficiency anemia is the most common nutritional deficiency in the country, ${ }^{3}$ and plant-based diets may contain more iron than other diets. Thus, habitual ingestion of a plant-based diet may lead to a low ingestion of some nutrients, especially some vitamins, and, in the poorest regions, a low ingestion of energy as well.

Plant-based diets like the diet typical of rural areas in Mexico are high in substances that may interfere with the absorption of several nutrients. Of relevance to this potential effect is a study ${ }^{4}$ in which we evaluated the effect of an average rural diet on the absorption of nutrients. The average rural diet contained $1.1 \pm 0.1 \mathrm{~g}$ of phytic acid per day and $40.2 \pm 2.6 \mathrm{~g}$ of dietary fiber per day. The absorption of nutrients with this diet was evaluated by balance studies using polyethylene glycol as a quantitative marker and it was compared with the absorption of the same nutrients with an average urban diet, which contained more animal foods. On average, the urban diet contained only $0.07 \mathrm{~g}$ of phytic acid per day and $12.1 \pm 0.9 \mathrm{~g}$ of dietary fiber per day. A summary of the results of this study is presented in Table 1. The apparent absorption of all nutrients studied was significantly reduced with the rural diet. In particular, there was a very large decrease in absorption of iron, zinc, and calcium with the rural diet. This study and the large body of information about the negative effect of plant food constituents on the absorption of minerals suggest that the low bioavailability of nutrients might be an important mechanism responsible for the high incidence of nutrient deficiencies in the population who habitually consume plant-based diets.

Many of the regions of Mexico where plant-based diets are habitually consumed are also regions where para- 